the examination was entirely macroscopic, neither a microscope nor the means for preserving and hardening the specimen being available. This is matter for regret, as it is possible that the existing lesions were not confined to the walls of the bloodvessels.

These two cases seem to me to supplement one another, and although it is as a rule unwise to generalise from single examples, we know so much of the symptomatology and of the morbid appearances that indicate cerebral con. cussion that we could well anticipate similar conditions in a corresponding injury to the spinal cord. In both events the spinal column was subjected to sudden and severe jar, and in both with a very similar and striking result. Admitting that the injury in the instance of the stag was an exceptionally severe one (for the contusing power of a ball travelling at a high rate of velocity is fully recognised), still in the case of A. C- there were certain circumstances which tended to render the blow he received one of great severity. He fell backwards from a train moving at fair speed in the opposite direction, receiving thus a considerable impulse; and when thus impelled he probably struck, judging from the localised nature of the bruise found, upon a projecting surface. Such conditions must have caused a sudden and severe stroke, and probably produced in him similar lesions to, but slighter than, those found in the stag.

Mr. Page, in his masterly and most valuable work upon "Injuries of the Spine and Spinal Cord," p. 19, refers to a case recorded by Dr. J. Liddell, in which a Minié ball striking $a$ man to the left of the second lumbar vertebra and emerging some inches to the right fractured in its passage its spinous process. Paraplegia and paralysis of the bladder were at once developed, but at the end of two months were being gradually recovered from. In this instance the circum. stances which gave rise to the injury were identical with those which occurred in the case of the stag, and I take it that the symptoms developed in the man owed their origin to such lesions in the spinal cord as were found to exist in that of the animal. Here, however, I would state my belief that even in the spinal cord we may have symptoms of $a$ passing "concusaion" in which probably the stroke being less severe, the harm actually done is neither so marked nor so grave. My reason for thinking this is that I have known a deer shot in the neck fall instantly and lie apparently dead for some little time, but, when approached, gather itself up with a visible efforb and go off at sufficient speed to lead to its escape. In it, I imagine, the ball passed immediately over the spinal column without perhaps actually injuring it, but sufficiently close to com. municate to it such a jar as for the time being inhibited the action of the organ it contained. What seems to render this more probable is the fact that a ball striking some other and non-vital part beyond, producing the sound of impact and the flight of the deer, would have afforded no immediate evidence of injury. ${ }^{2}$ Even, however. in these lighter shocks-these so-called "concussions" - there is probably always a marked lesion existing. That this is usually so necropsies in fatal cases of cerebral concussion reveal to us, and in these the appearances found are precisely those which I have described as occurring in the spinal cord I examined. They are the coarse evidence of the jar the nervous mass has sustained, and the violence of which, so far as the naked eye carries us, seems to fall largely upon its vascular apparatus. Whether this be due, as Duret holds, to the result of force communicated to the cerebro-spinal fluid and its effects in the peri-vascular spaces, or to the vibratory thrill imparted to the mass itself, matters little ; in either case what occurs is an apparently temporary, though happily incomplete, suspension of its essentially vital action. That this is the direct issue of the shake, and is independent of such vascular lesions as post-mortem examination reveals, is, I consider, probable; for although these mark well and emphatically the severity and nature of the injury sustained, they are, as a rule, insufficient per se to have produced a fatal result. That such lesions exist to a greater or less extent in all cases of cerebral and spinal concussion which do not die wellnigh instanta. neously is my belief, and they write the record that the notable disturbance of function observed depends upon organic injury. I have some doubt, however, that such evidence can never be absent, and it comes from the

2 In this reasoning I am not forgetful of the "knock-down blow" from general shock, familiar alike to the army surgeon and to the sportsman, which is apt to follow the blow of a missile, but where the recovery is, as a rule, rapid. observation of cases of cerebral concussion seen at the moment the injury was received. I vividly remember the case of one man-certainly the most striking in my experience-whom I saw fall from a height of some fourteen feet and alight upon his vertex in the hard roadway. The appearance be presented was very impressive : the terrible pallor of his face, the dropped jaw, the fixed pupil and insensitive eye, the arrested breathing, and the absence of all pulse at the wrist, made me believe he was dead. It was only when having bared his chest and put my ear over his heart, I could detect such far away and feeble sounds as mark generally a rapidly ebbing life that I recognised that vitality was not quite extinct. Under the use of stimulants applied chiefiy externally he slowly rallied, and ultimately recovered. But my reflection was this : Suppose that man had diedas in the presence of a heart with feeble walls or one less stable in its ennervation, and in the absence of help, he probably would, - what then would have been the appear ances presented by his brain? If, as the experiments of the most recent observers seem to show, a primary anæmia from arterial spasm, the result of a powerful peripheral irritation, be the primary step in the development of the phenomena of concussion, and a congestive stage the sequel to this, it is, I think, probable that the blood extravasations so generally found can only occur after vascular relaxation; and that, if cerebral shock be immediately fatal, a singularly pale brain with great turgidity of its veins may alone indicate the mode of dying. Such a belief is contrary to the teaching of the highest anthorities, and it may be groundless; but it is just possible that it may explain the absence of all coarse cerebral lesions in certain cases. attached to the records of which are the names of generally reliable observers.

The occurrence of concussion of the brain, aparb from marked injury to its bony case, has always been admitted, but it has been notably otherwise with concussion of the spinal cord. The experience which prompts this note proves, I think, that such an injury may occur without important structural injury of the vertebral column, thas it is the result of severe and direct injury, that its $85 \mathrm{~m}$. ptoms are at once apparent, and that when they are present there is some easily recognisable lesion producing them. The further teaching is this, that the treatment of such cases should be that usually followed in those of cere. bral concussions, the main indications being the pursuit of such measures primarily as will prevent the further effusion of blood from ruptured vessels, the prompt relief of that state of active congestion which, as in cases of cerebral concussion, may possibly supervene, and the allowance of such a period of rest as will enable nature to repair the important injuries the cord has certainly sustained.

Cannes, France.

\section{A SUCCESSFUL CASE OF}

\section{ILEO - SIGMOIDOSTOMY (SENN'S METHOD)}

\section{FOR INTESTINAL OBSTRUCTION DUE TO} MALIGNANT DISEASE OF THE HEPATIC FLEXURE OF THE COLON;

WITH SOME REMARKS ON INTESTINAL ANASTOMOSIS AND A DESCRIPTION OF A MODIFICATION OF SENN'S BONE PLATES.

BY H. LITTLEWOOD, F.R.C.S.,

HON. ASSISTANT SURGEON TO THE GENERAC INFIRMARY AT LEEDS.

THE following are the notes of the case: $G_{\text {. }} F_{-}$, aged thirty-five, was admitted to the Leeds Infirmary on March 17th, 1891, under the care of Mr. W. H. Brown, with marked symptoms of intestinal obstruction. Thepatient said that since Christmas he had had a good deal of pain in the right iliac region, and for the last three weeks he had been unable to work. Three days before admission vomiting commenced, and continued at intervals; no motion or flatus was passed during this interval. On admission the patient was very $\mathrm{ill}$, suffering from severe spasmodic attacks of abdominal pain and frequent vomiting. The abdomen was distended. The intestines could be seen distended and

1 Paper read before the Leeds and West Riding Medico-Chirurgical Society on Dec. 4th, 1881. 
noving beneath the abdominal wall. The whole of the right side of the hypogastrium was dull. Before admission the patient had a small dose of morphia. Soon after admission the bowels were well opened. An enema was given, followed by another good evacuation. After this the patient was much better, vomiting and pain ceased. He left the infirmary on March 17 th.

He was admitted again on Aug. 14th with well-marked intestinal obstruction. At no time since leaving the infirmary had he felt really well. Hehad had occasional attacks of vomiting and constipation, of ten complaining of pain after food. He had seldom been able to work more than two or three days at a time. Latterly the attacks had increased in frequency and severity. The bowels had not been opened 'for the past five days; he passed flatus a few times during this period, and vomited frequently. On admission the following note was made :- "Patient looking very ill; not much collapsed. Temperature $984^{\circ}$; pulse 100 . Abdomen distended; resonant, Except low down in the flanks, coils of distended intestine can be easily seen moving (gometimes violently) through the abdominal parietes at frequent intervals. The movements can be easily excited on palpating the abdomen. No tumour can be seen or felt in any part of the abdomen. Complains of a griping intermittent pain, radiating all over the abdomen. Between the attacks of pain there is merely a feeling of distension." Since admission he has vomited several times; the vomit has not been feculent at any time. He has not passed any blood or pus per anum. Nothing felt by rectal examination. Soon after admission I saw the case with Mr. W. H. Brown, sunder whose care the patient had been admitted, and we agreed that as enemata had answered so well on a former occasion they should again be tried. Mr. Brown, going away the next day, handed the case over to my charge. A gravitation enema of a pint of warm olive oil was given; this was soon followed by a good evacuation, after this a simple enema was given, with good results.Aug. 15th : Feels much more comfortable; pain and disten. sion much less; vomiting has ceased.-19th: Has been feeling more comfortable; still some distension of abdomen. No vomiting; bowels opened by enemata -23rd: During last few days patient has not been quite so comfortable. Some feeling of nausea; bowels opened by enemata. Abdomen still distended; patient has a feeling of general abdominal discomfort. Sometimes movements of the bowels can be seen, and to.day can be easily excited by abdominal palpation. At a consultation with the majority of the surgical staff the general opinion expressed was that the case was one of intestinal obstruction due to malig. nant disease of the colon, probably at the upper part of the sigmoid flexure. Abdominal exploration was advised, with a view of removing the growth if possible; if not, to stitch the bowel above the obstruction to a part of the collapsed bowel below.-24th: The patient being ander the influence of ether, with the assistance of $\mathrm{Mr}$. Moynihan, the resident surgical officer, I operated by making an incision three inches long in the middle line between the umbilicus and pubes, going through the edge of the right rectus. All bleeding having been stopped, the peritoneal cavity was opened to the same extent. There was some fluid in the peritoneal cavity. The small intestines were very much distended, some parts of the ileum being as much as two inches in diameter. They were injected. Creum and ascending colon distended. Transverse, descending colon, and sigmoid flexure collapsed. A large mass of growth about the size of an orange was found situated in the hepatic flexure and fixed. Mr. Mayo Robson, who was present at the operation, af er having examined, agreed with me as to the inadvisability of attempting removal, and thought with me the best plan of treating the case would be to stitch the lower part of the ileum to the upper part of the sigmoid flexure. This I did by first pulling 2 loop of ileum out of the abdominal wound, emptying it of its contents, then surrounded it with a piece of indiarubber tubing. The same was done to a loop of sigmoid flexure. An incision about one inch long was made in the convex surface of each. Senn's decalcified bone-plates were inserted, and secured in the usual way. Four extra silk sutures were then put in round the margin of the plate, on the convex surface of the two pieces of bowel, to givealittleextra security. The bowels were then replaced in the abdominal cavity, the cavity carefully wiped out, and the abdominal wound stitched up in three layers-peritoneum, muscles and fascia, and skin. - 26 th : Patient feels very well. Has passed flatus; no

romibing ; temperature normal; less distension of abdomen.-28th : Patient still feels very well. Had bowels noved to-day. Sits up in bed.-Sept. 1st: Has improved since last note. Dressed to-day, wound quite healed; no distension of the abdomen. One of the bone-plates has passed per anum to-day.-5th : Still improving; bowels acting regularly. - 7th : Patient feels very well; has been up to-day-i.e., fourteen days after operation,-10th : Improvement maintained; appetite good. -12th : Says he feels perfectly well. - 16th : Left infirmary to-day-i.e., twentythree days after operation. Temperature $100 \cdot 2^{\circ}$ on the mornings of the $25 \mathrm{th}$ and $26 \mathrm{th}$. Temperature $99 \cdot 4^{\circ}$ on the evening of the $27 \mathrm{th}$, and after that normal.

Diet.-For the first five or six days after the operation he was fed chiefly on milk and Benger's food. Had fish on Aug. 31st, chicken on Sept. 3rd, chop on Sept. 4th, ordinary diet on Sept. 7th. Patient was shown at the meeting of the Leeds and West Riding Medico-Chirurgical Society on Oct. 9th; was apparently very comfortable; no abdominal distension; bowels opened every day. A few days after this he went to work as a miner.

The case described above is an additional demonstration

FIG. 1.

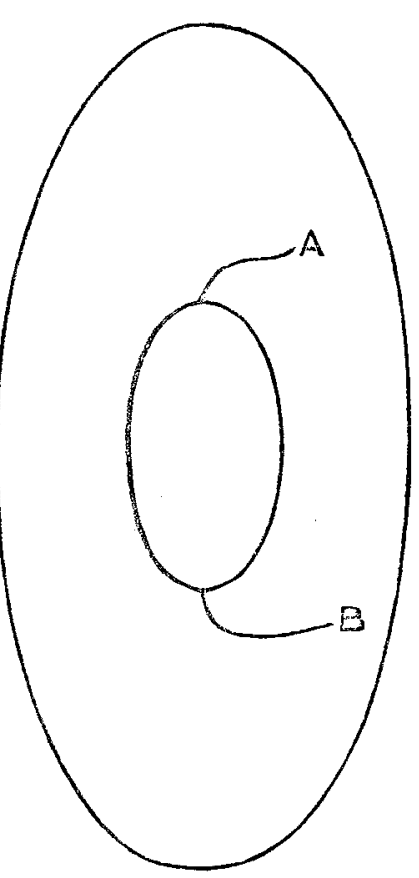

FIG. 3.

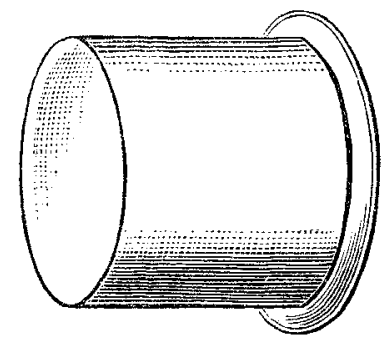

to the cases already recorded of the possibility of treating intestinal obstruction by establishing a communication between the distended bowel above the obstruction and the collapsed bowel below, and so cutting off the obstructing portion from the intestinal tract. We owe to Senn an enormous debt for this great advance in the treatment of intestinal obstruction, and, considering his paper was read in September, 1887, at the International Medical Congress at Washington, it is very remarkable that up to the present time only four cases have been recorded of this method having been used for the treatment of intestinal obstruction. Two were performed by Senn, one by Mr. F. B. Jessett, and the other by Mr. Reeves. They are referred to in a letter by Mr. Jessett in THE LANCET of Feb. 21st, 1891. Similar operations have been performed for other causes in several cases-e.g., injuries, pyloric obstruction, \&c. This case differs somewhat from the other recorded cases in that the intestine was not divided, but a simple lateral anastomosis made. In Senn's two cases the growth was 
first excised; in the other two the operation of lateral implantation was performed; all of them necessibating a division of the intestine.

Before we were acquainted with this method, the treatment of cases of intestinal obstruction requiring surgical interference might have been arranged into three classes :1. Those in which some definite obstruction was found and was relieved by operation. 2 Cases in which the obstruction was found and could not be removed, an artificial ann being made. 3. Cases in which, after a prolonged search, either the obstruction was or was not found, the patien dying of shock. It is for the treatment of such cases as occur in Classes 2 and 3 that Senn's method offers such advantages. In Class 2 the artificial anus is obviated. In cases belonging to Class 3 , if the obstruction cannot be found after a reasonable time has been expended in the search, then lateral anastomosis offers the best chances of a successful termination. From assisting at a large number of cases of abdominal sections, it has always struck me that time is a very important element in the success of a case. By this method there is a great saving of time. With a little practice on the cadaver, or having seen or assisted at the operation, it can very quickly be performed (in about ten or fifteen minutes). 1 have ventured to suggest a modification of Senn's plates, with the idea of (1) doing away with the four stitches attached to the upper and lower margins of the apertures of the plates, which perforate the whole thickness of the intestinal walls; (2) of performing the operation more quickly; (3) of ensuring a good opening between the two pieces of intestine. The plates have been made for me by Messrs. Maw, Son, and Thompsrin; the accompanying drawing is by Mr. Haigh. The suggested modification is to fix a tube of decalcified bone (Fig. 3) into the aperture of one of the plates (Fig. 2). This should be made to accurately fio into the aperture of the other (Fig. 1); by this method the two plates could be held together, and the two parts of the intestinal walls between them brought evenly into contact with each other. It might be well to have a piece of fine silk attached to each of the ends of the apertures (as marked $A$ a, $B$ b in Figs. 1 and 2 ), so that by tying $A$ and $\mathrm{B} b$ together greater security would be made. The intestinal walls around the margins of the plates should be attached by a few sutures.

In a very interesting paper by Dr. Halsted of Baltimore a method of lateral apposioion by sutures is described. He insists very strongly on the importance of including some of the submucons layer of the intestinal wall in these and all suturing operations of the intestine. I am sure it is very important to recognise this observation, but cannot help thinking the bone plates will have the advantage over any method of suturing, owing to their simplicity and the time aaved in using them.

Leeds.

\section{Clinital a}

\section{MEDICAL, SURGICAL, OBSTETRICAL, AND THERAPEUTICAL.}

\section{THE NASO-PHARYNX IN INFLUENZA.}

\section{By Arthur E. Nevins, F.R.C.S. Edin.}

SINCE the influenza appeared in Earope a couple of years ago there have been abundant opportunities for everyone to become acquainted with the symptoms peculiar to this disease, and it has been generally recognised that catarrh of the nasal passages, with a more or less free evacuation of mucous, is a very common symptom. Many cases, how. ever, come under observation in which there is comparatively little muccus discharge from the nose. But in these cases also there is always, so far as my observations go, a severe inflammation of the whole mucous lining of the naso-pharynx, which is often more intense, and productive of more acute distress to the patlent than in the cases in which the catarrhal symptoms are more obvious. It seems, in fact, as if it was a rule, almost without an exception, that in influenza the infection first attacks the naso-pharynx, and it is the intense inflammation of this mucous membrane (which, when als) its ramifications through the ethmoidal and frontal sinuses are taken into account, represents a very large superficia? area) that is the cause of the intense deoility and grave constitutional disturbances that ultimately supervene. The sbock to the system is quite comparable to that of $a b$ extensive burn or a rapidly spreading attack of erysipelas The appearance of the tonsils and pharynx in many cases quite bears out this idea, for the mucous membrane is seev to be of a deep red colour, ratber dry, and from its puffiness suggestive of subjacent cellulitis. Nasal respiration is impeded, and there is a heavy, unpleasant smell, which is very perceptible to the patient, and also to those about him.

The intense cardiac and general enfeeblement which accompanies a "hospital throat" is well known; to many members of the profession it has been a painful personal experience. The prostration in cases of influenza is very similar in character, and probably dependent upon the same cause. If this is the case, it would lead us to look to local remedies, applied to the inflamed naso-pharyngeal mucous membrane, as likely to be efficacious in treating the general condition. The object of the present note is to degcribe a simple line of treatment, based upon a belief in this theory, which has been singularly successful in every case of influenza that has come under my own observation. furthermore, I can testify from personal experience to the immediate relief which it gives to the headache, feverish. ness, rigors, and other discomforts of the acute stage of an attack. It consists of spraying the nostrils and throat with a lotion composed of four grains of chlorate of potassium, four minims of tincture of perchloride of iron, to one ounce of peppermint water. The patient leans his head well back, and the spray is sent ap one nostril untilit can befelt trickling in to the throat. In about twenty to thirty seconds a free expec toration of tenacious, viscid mucus takes place. The other nostril is similarly treated, and then the throat is sprayed, While this is being done the patient should inhale deeply, as by that means the spray is carried down into the larynx and trachea, which is important, as these parts are often affected by the inflammation. The relief and comfort which immediately follow the application is remarkable, and decrease in the fever is soon noticeable. The patient is able to take some liquid food without retching after it, and as a rule sleep soon follow. If the treatment is repeated atintervals of two hours for the first twelve hours, and of four hours during the next twelve hours, it will be usually found that the temperature is by that time nearly normal, and that convalescence has fairly commenced. As regards general treatment nothing need be said here; it must be adapted to the special circumstances of each case. There are, however, two lines of treatment which should in most cases be avoided. Inasmuch as intense cardiac enfeeble ment is very common in these cases, the antipyretic drugs, which are all-powerful cardiac depressants, are contra indicated. As a rule, purgatives also are undesirable, for it is very common to find some degree of inflammation of the crecum or colon as a complication in these cases. Hot applications to the abdomen are much more beneficial, as a rule. In conclusion, it may be well to mention that if a spraying apparatus is not at hand, the same effect can be got by pouring about twenty drops of the lotion up the nostril with a teaspoon and making the patient gargle the throat afterwards, but the spray is the more com. fortable mode of application. Hanley, Staffs.

\section{NOTE ON PRICKLY HEAT,}

\section{B Y J. A. WETHEREL, M. D.}

UNDER the term "lichen tropicus"must be embraced three primary lesions, in frequency taking the following order :1. A vesicular eruption, the vesicle, of the size of a pin's head, elevated on a roseolons, inflammatory base, being filled with colourless perspiration; sometimes arranged in segments of rings, at others diffusedly spread. On a superficial examination, it resembles much the efflorescence of measles or scarlatina, and occurs principally on the nape of the neck, the flexor surfaces of the forearms and legs, and the front of the abdomen. Even habitants for many years of semi-tropical regions are apt to exhibit this form, especially those who wear woollen underclothing. 2. A wheal with a red surrounding halo, accompanied by intense itching; 